\title{
OSCILLATION THEOREMS OF ARITHMETICAL FUNCTIONS
}

\author{
BY \\ EMIL GROSSWALD( $\left.{ }^{1}\right)$
}

1. Introduction. In 1853, Tchebycheff, in a letter to Fuss (see [30]), made the statement that there are more primes congruent to $3(\bmod 4)$, than primes congruent to $1(\bmod 4)$. With this assertion, Tchebycheff seems to have ushered in the complex of problems that has come to be known as "comparative prime number theory" in the terminology of Knapowski and Turán (see [11]). This complex of problems itself is part of a more comprehensive theory dealing with the oscillations of arithmetical functions representable in the form $S(x)=\sum_{n \leqq x}^{\prime} a_{n}$ (see $\S 5$ ). In the study of the oscillatory properties of these functions one frequently encounters infinite sets $X=\left\{x_{n}\right\}$, with real $x$ and $\lim _{n \rightarrow \infty} x_{n}=+\infty$. Any infinite set with this property will be called a set of type $X$, or simply an $X$-set. Sometimes it is possible to select $X$-sets in such a way that all their elements are natural integers; such $X$ sets will be called $Z$-sets. For each $X$-set one can define its "counting function" $W(y)=W_{X}(y)=\sum_{x_{n} \leqq y: x_{n} \in X} 1$. Often one is interested in the order of magnitude of $W(y)$; for instance, one may inquire whether, for a given $X$-set,

$$
\limsup _{y \rightarrow \infty} W(y) / \log y>0
$$

does, or does not hold. If this inequality holds, one often expresses it by the symbol $W(y)=\Omega(\log y)$.

Let $S(x)$ be an arithmetical function, $\lim _{x \rightarrow \infty} S(x)=+\infty$, and suppose that, for $x \rightarrow \infty, S(x)=f(x)(1+o(1)$ ), where $f(x)$ is a known (often-but not alwaysfairly simple, even holomorphic) function. Then the study of the (often very complicated) function $S(x)$ is reduced to that of the difference $R(x)=S(x)-f(x)$. Concerning $R(x)$, one tries to establish results of one of the following two types:

(a) $R(x)=O(g(x))$, called $O$-theorems; and

(b) $R(x)=\Omega(h(x))$, called $\Omega$-theorems.

Here again, $g(x)$ and $h(x)$ are known (usually fairly simple) positive functions.

Presented to the Society, April 4, 1966 under the title Oscillations of arithmetical functions; received by the editors April 15, 1966.

(1) The author gratefully acknowledges his indebtedness to Professor H. Wilf for many stimulating conversations. This paper was completed while the author was on a sabbatical leave at the Institute Henri Poincaré, Paris (France) with partial support from the National Science Foundation through the Grant NSF-GP-3137. 
Whenever possible, one tries to improve (a) to

$\left(\mathrm{a}^{\prime}\right)|R(x)| \leqq C g(x)$, with a known constant $C$; and (b) to

(b') $R(x)=\Omega_{ \pm}(h(x))$,

or even to the stronger statement

$\left(\mathrm{b}^{\prime \prime}\right)$ For all $C \leqq C_{0}\left(C_{0}>0\right.$, a computable constant), the two inequalities $R(x)$ $>C h(x)$ and $R(x)<-C h(x)$ hold each on a (naturally, different) $X$-set (or Z-set). In order to simplify the writing, we shall often abbreviate a statement like $\left(b^{\prime \prime}\right)$ by $R(x) \gtrless \pm C h(x)$.

Returning for a moment to Tchebycheff's assertion, its most naïve interpretation (which need not be that of Tchebycheff) is equivalent to the statement that

$$
\pi(x ; 4,3)-\pi(x ; 4,1) \geqq 0
$$

holds at least for all sufficiently large $x$. Here, and in what follows, $\pi(x ; k, a)$ stands for the number of primes $p \leqq x$, satisfying $p \equiv a(\bmod k)$.

Hardy and Littlewood have shown (see [4]) that $\left(^{*}\right)$ is false. In fact, if we denote the first member of $\left(^{*}\right)$ by $R(x)$, then Hardy and Littlewood have proved( $\left(^{2}\right)$ that there exists a $C>0$, such that $R(x) \gtrless \pm C x^{1 / 2} \log \log \log x / \log x$, each inequality being verified on an $X$-set. This result of comparative prime number theory is formulated here in the form $\left(b^{\prime \prime}\right)$ above and illustrates the previous assertion that the results of comparative prime number theory can be formulated as statements concerning oscillations of arithmetical functions.

2. Historic survey. Some important $\Omega$-theorems were obtained almost immediately after the proof of the Prime Number Theorem. Among these one may quote, for instance, the following:

In 1898, E. Phragmén [26] proved a conjecture of Tchebycheff stating that to every $\delta>0$ there exists an $X$-set on which

$$
\left|\{\pi(x ; 4,3)-\pi(x ; 4,1)\} x^{-1 / 2} \log x-1\right|<\delta .
$$

In 1899, J. L. W. V. Jensen [10] asserts that $\pi(x)-\operatorname{li} x=\Omega\left(x^{1 / 2-\varepsilon}\right)$, where $\pi(x)=\sum_{p \leqq x} 1$ and li $x=\int_{2}^{x}(\log y)^{-1} d y$. No proof is offered by Jensen. (It may be worthwhile to recall also that in the same paper one finds the assertion that the author has proven the Riemann hypothesis.)

In 1901, H. von Koch shows (see [21] and [22]) that, on the Riemann hypothesis, $\pi(x)-\operatorname{li} x=O\left(x^{1 / 2} \log x\right)$. This result is used by E. Schmidt (see [29]), who proves the following: Let $\zeta(s)=\sum_{n=1}^{\infty} n^{-s}(s=\sigma+i t, \sigma>1)$, be the Riemann zeta function, define $\theta=\sup \{\sigma \mid \zeta(\sigma+i t)=0\}$ and set $f_{0}(x)=\sum_{p^{m} \leqq x} m^{-1}=\sum_{m \geqq 1} m^{-1} \pi\left(x^{1 / m}\right)$; then, for all $\lambda<\theta$, and arbitrarily large constant $C>0, f_{0}(x)-$ li $x \gtrless \pm C x^{\lambda}$, each inequality

$\left(^{2}\right)$ [4] contains only a sketch of the proof, which does not seem to have ever been published in full by Hardy and Littlewood. 
holding on some $X$-set; furthermore, each of the inequalities

$$
f_{0}(x)-\operatorname{li} x \gtrless \pm(1 / 29) x^{1 / 2}(\log x)^{-1}
$$

holds on an $X$-set.

In his important paper [23] of 1905, among many other results Landau gives also a proof of the fact that setting $\Psi(x)=\sum_{p \leqq x} \log p$, both inequalities $\Psi(x)-x \gtrless 0$ hold on $X$-sets. While this statement is essentially equivalent to one of E. Schmidt, Landau's proof is noteworthy on account of his method.

In 1914, Littlewood ([25]; see also [4]) proved the important result that there exists a positive constant $C$, such that both inequalities

$$
\Psi(x)-x \gtrless \pm C x^{1 / 2} \log \log \log x
$$

hold, each on an $X$-set, or, with an obvious notation, $\Psi(x)-x=\Omega_{ \pm}\left(x^{1 / 2} \log _{3} x\right)$; equivalently, $\pi(x)-\operatorname{li} x=\Omega_{ \pm}\left(x^{1 / 2}(\log x)^{-1} \log _{3} x\right)$.

In 1930, Pólya [27] gave a lower bound for the number of sign changes $W(y)$ of $\Psi(x)-x$, when $x$ runs through the interval $1 \leqq x \leqq y$, as follows: with $\zeta(s)$ and $\theta$ defined as before, let

$$
P=\{s=\theta+i t \mid \zeta(s)=0\}
$$

if $P=\varnothing \quad\left(\varnothing=\right.$ the empty set), set $\gamma=+\infty$, otherwise $\gamma=\min _{\theta+i t \in P}|t|$. Then $\lim \sup _{y \rightarrow \infty} W(y) / \log y \geqq \gamma / \pi$.

3. Purpose and summary of the paper. In spite of these and other successes, until recently the general progress in this field was rather slow, as pointed out in $[11$, p. 299]. Also, each problem was usually handled by methods tailored specifically for its solution. The situation changed when Turán proved his important theorem (see [32]), giving upper and lower bounds for sums of the form $\sum_{n=1}^{N} a_{n} z_{n}^{v}$, where $a_{n}$ are fixed numerical constants, $z_{n}$ are given complex numbers and where $\nu$ may be selected within certain ranges. It turned out that this "new method in analysis" permits a systematic treatment of a large range of oscillation problems. In particular, it permits to prove localization theorems, i.e., to determine not only whether a certain function $R(x)$ changes sign for arbitrarily large values of $x$ (that is, on an $X$-set), but also to find lower bounds for the frequency of sign changes and upper bounds for the first change of sign.

It was perhaps due to the success of this method, that other approaches to this complex of problems have been somewhat neglected. It is the purpose of this paper to indicate one such alternative approach, based on ideas that go back to Landau [23] and Pólya [27]. The method has already been used occasionally in the study of specific problems (besides Landau and Pólya, see especially Ingham [9]), but, as far as I know, not for a large variety of problems and, in particular, not in connection with comparative prime number theorems. The present method is weaker than Turán's method, as far as localization theorems are concerned, but 
it has the advantage of an almost automatic application. Therefore, while many results obtained in this paper are already known, and many of the others could be obtained, presumably, also by Turán's method, there is some hope that the present approach will permit generalizations to situations where the "new method in analysis" is not directly applicable and not easy to be made effective. On the other hand, even in the proof of known results, the esthetic appeal due to the simplicity and the unified approach of the present method seems to justify its use.

The theorems and corollaries needed are listed in $\$ 4 . \$ \$ 5$ and 6 contain the general scheme for the proofs of oscillation theorems by the present method and some comments. In $\S 7$ the method is used to obtain the (known) oscillation theorems for $\Psi(x)-x, f_{0}(x)-$ li $x$ and $\pi(x)-$ li $x$. $\S 8$ discusses the bearing of the present method on some conjectures known by the names of Mertens, Pólya and Turán. $\$ \S 9$ and 10 contain two (possibly new) results concerning $\Omega$-theorems, for the oscillations of the functions $S(x)=\sum_{n \leqq x}^{\prime} 2^{\omega(n)}$, and $S_{m}(x)=\sum_{n \leqq x}^{\prime} c_{n}(m)$, where $\omega(n)=\sum_{p / n} 1$ and $c_{n}(m)=\sum_{(k, n)=1 ; k \bmod n} \exp (2 \pi i k m / n)$ (Ramanujan sum), respectively. $\$ \$ 11$ to 14 treat some problems of comparative prime number theory, such as the oscillations of the differences $f_{0}(x ; k, a)-f_{0}(x ; k, b)$ and $\pi(x ; k, a)-\pi(x ; k, b)$; here $\pi(x ; k, a)$ has been defined before and $f_{0}(x ; k, a)=\sum_{p^{m}}^{\prime} \leqq x ; p^{m} \equiv a(\bmod k) m^{-1}$. Finally, in $\S 15$ it is shown that for certain values of $k$, the difference $\pi(x ; k, a)-\pi(x ; k, b)$ indeed changes its sign infinitely often.

\section{Some theorems and corollaries.}

THeOREM A (LANDAu [23]). Let $f(x)$ be real and positive for real $x \geqq x_{0}$ and suppose that $\int_{x_{0}}^{\infty} f(x) x^{-s} d x$ has the finite abscissa of convergence $\theta$; then the function $F(s)$ represented for $\sigma>\theta$ by the integral, has a singularity at $s=\theta$.

Corollary A. If $F(s)=\int_{x_{0}}^{\infty} f(x) x^{-s} d s$, with $f(x)$ as in Theorem A, the integral being convergent in some half-plane and $F(s)$ is holomorphic for $\sigma>\theta$, but not in any half-plane $\sigma>\theta-\varepsilon$ with $\varepsilon>0$, and if furthermore $s=\theta$ is a point of regularity for $F(s)$, then $f(x)$ changes sign at all points of an infinite set $X=\left\{x_{n}\right\}$, with $\lim _{n \rightarrow \infty} x_{n}$ $=+\infty$ (i.e., at all points of an $X$-set).

The corollary is stated in almost the same terms in [27] and follows immediately from the theorem.

TheOREM B (Pólya [27]). Suppose that $f(x)$ and $F(s)$ are as in Corollary A; furthermore, suppose that $\varepsilon_{0}>0$ is such that $F(s)$ is meromorphic for $\sigma \geqq \theta-\varepsilon_{0}$. Denote by $P$ the (possibly empty) set of poles $s=\theta+$ it of $F(s)$, of abscissa $\sigma=\theta$; set $\gamma=\min _{\theta+i t \in P}|t|$ if $P \neq \varnothing, \gamma=+\infty$ if $P=\varnothing$. Then $W(y)=\sum_{x_{n} \leqq y: x_{n} \in X} 1$ satisfies $\lim _{y \rightarrow \infty} \sup W(y) / \log y \geqq \gamma / \pi$, where $X$ is the $X$-set of sign changes of $f(x)$.

REMARK. If $\gamma=0$, Theorem B asserts nothing and if $\gamma \neq 0$, Corollary A guarantees the existence of the $X$-set of sign changes. 
It has been pointed out repeatedly in the literature (see, e.g., [8] or [11]) that Pólya's proof of Theorem B in [27] contains a gap. Actually, the gap affects only the case $\gamma=+\infty$, which is rarely invoked. However, the statement of the theorem is correct $\left({ }^{3}\right)$ even if $\gamma=+\infty$.

THEOREM C (SEE [3]). Let $f(x)$ be real for real $x \geqq x_{0}$ and satisfy also the following condition: $\int_{x_{0}}^{\infty} f(x) x^{-s} d x$ converges for $\sigma \geqq \sigma_{0}$ and represents there a function $F(s)$, holomorphic for $\sigma>\theta$ and with $\lim _{\sigma \rightarrow \theta^{+}} F(\sigma)=+\infty$. If there exists a $t_{0} \neq 0$ such that

$$
\lim _{\sigma \rightarrow \theta^{+}} \sup \left|F\left(\sigma+i t_{0}\right) / F(\sigma)\right|=a, \quad \text { with } a>1,
$$

then $f(x)$ changes signs at all points of an $X$-set; the same conclusion holds if $a=1$, provided that also

$$
\lim _{\sigma \rightarrow \theta^{+}} \sup \left\{\left|F\left(\sigma+i t_{0}\right)\right|-|F(\sigma)|\right\}=+\infty
$$

It is sometimes convenient to reformulate Theorem C. As a motivation, let us observe that if $f(x)$ satisfies certain additional conditions of regularity, then much more is actually true. If we set $f_{\sigma}(x)=f(x) x^{-\sigma}$ for $x \geqq x_{0}, f_{\sigma}(x)=0$ for $0 \leqq x<x_{0}$, $y=\log x$ and $e^{y} f_{\sigma}\left(e^{y}\right)=g_{\sigma}(y)$, then $F(\sigma+i t)=\int_{-\infty}^{\infty} g_{\sigma}(y) \exp (-i y t) d y$, the Fourier transform of $g_{\sigma}(y)$. If, for instance $g_{\sigma}(y) \in L(-\infty,+\infty)$ then $\lim _{t \rightarrow \infty} F(\sigma+i t)=0$ by the Riemann-Lebesgue theorem; if, furthermore, also $g_{\sigma}(y) \geqq 0$ (but not $g_{\sigma}(y)=0$ a.e.), then $\lim _{t \rightarrow \infty} F(\sigma+i t) / F(\sigma)=0$. The present situation is, of course, not quite the same. Indeed, the functions $g_{\sigma}(y)$ that we shall want to consider are not, in general, Lebesgue integrable over $(-\infty,+\infty)$, at least not for $\sigma=\theta$; they also fail to be either continuous, or monotonic (these properties are used to prove strong versions of Theorem C; see [3]). For these reasons, it does not seem likely that Theorem $\mathrm{C}$ could be replaced by a simple and at the same time much stronger statement. Let us consider, however, the following

Definition $\left({ }^{4}\right)$. Let $h(\sigma)$ be a positive, real-valued function, defined for $\sigma>\theta$. Let $T=\left\{t_{n}\right\}$ be a (finite, or infinite) set of real numbers. Let $f(x)$ and $F(s)$ be defined as in Theorem $\mathrm{C}, f(x)$ satisfying the condition of the theorem. Then we shall say that $(x)$ belongs to the class $K_{h, T}$ if the conditions

$$
\lim _{\sigma \rightarrow \theta^{+}} \sup \left|\frac{F\left(\sigma+i t_{n}\right)}{F(\sigma)} h(\sigma)\right|=a_{n} \quad\left(a_{n}>1\right)
$$

for all $t_{n} \in T$ imply that $f(x)$ changes signs on an $X$-set.

With this terminology and denoting the function $h(\sigma) \equiv 1$ simply by 1 , Theorem C becomes:

THEOREM $\mathrm{C}_{1}$. All functions $f(x)$ satisfying the conditions of Theorem $\mathrm{C}$ belong to the class $K_{1,\left\{t_{0}\right\}}$. proof.

$\left({ }^{3}\right)$ Professor Pólya kindly supplied in a letter the sketch for a complete (unpublished)

$\left.{ }^{4}\right)$ A referee's suggestion helped to improve this definition and is gratefully acknowledged. 
Corollary C (SEE [3]). Let $f(x)$ satisfy the condition of Theorem C; then $f(x)$ changes signs on an $X$-set, provided that $F(s)$ shows any of the following types of behavior:

(i) $F(s)$ has a pole at $s=\sigma+$ it $(t \neq 0)$, while $\lim _{\sigma \rightarrow \theta^{+}}(\sigma-\theta) F(\sigma)=0$;

(ii) $F(s)$ has a pole at $s=\sigma+$ it and a pole of lower order at $s=\sigma$;

(iii) $F(s)$ has poles of equal orders at $s=\sigma+i$ and at $s=\sigma$, with principal parts $\sum_{n=1}^{k} a_{n}(s-\theta-i t)^{-n}$ and $\sum_{n=1}^{k} b_{n}(\sigma-\theta)^{-n}$, respectively, with $a_{n}=b_{n}$ for $g \leqq n \leqq k$ and $\left|a_{g-1}\right|>\left|b_{g-1}\right|$, where $2 \leqq g \leqq k-1$ and $g=k+1$ means $\left|a_{k}\right|>\left|b_{k}\right|$;

(iv) $F(s)=c_{1} \log (s-\theta)^{-1}+c_{2} \log (s-\theta-i t)^{-1}+\phi(s)$, with $\phi(s)$ holomorphic for $\sigma \geqq \theta$ and with $t \neq 0, c_{1}, c_{2}$ real and $\left|c_{2}\right|>\left|c_{1}\right|$.

THEOREM D (SEE [3]). Let $f(x)$ be real for $x \geqq x_{0}$ and suppose that the integral $\int_{x_{0}}^{\infty} f(x) x^{-s} d x$ converges at least in some half-plane $\sigma \geqq \sigma_{0}$ and represents there a function $F(s)$ with the following properties:

(i) $F(s)$ is holomorphic for $\sigma>\theta$, but not in any half-plane $\sigma>\theta-\varepsilon$ with $\varepsilon>0$;

(ii) there exists a denumerable set $S=\left\{s_{n}=\sigma_{n}+i t_{n}\right\}$ without finite limit point, satisfying $\theta-\varepsilon_{0}<\sigma_{n} \leqq \theta$ for some $\varepsilon_{0}>0$ and such that $F(s)$ can be continued as $a$ meromorphic function in the open set $D$, obtained from the half-plane $\sigma>\theta-\varepsilon_{0}$ by making the cuts $s=\sigma+i t_{n}, \theta-\varepsilon_{0}<\sigma \leqq \sigma_{n}$;

(iii) for $s \rightarrow s_{n}(s \in D), F(s)=P_{n}\left(s-s_{n}\right) \cdot \log \left(s_{1}-s_{n}\right)^{-1}+F_{n}(s)$, where $F_{n}(s)$ is holomorphic at $s=s_{n}$ and $P_{n}(u)$ is a polynomial in $u$, of (effective) degree $k_{n}$, with $0 \leqq k_{n} \leqq k-1$, where $k$ is an integer depending only on $F(s)$ (in particular, $k$ is independent of $n)$.

Let $P_{1}=\left\{s_{m}=\sigma_{m}+i t_{m}\right\}$ be the set of poles of $F(s)$ with $\theta-\varepsilon_{0}<\sigma_{m} \leqq \theta$ and denote by $P$ the (possibly empty) set $\left\{s=\theta+i t \mid s \in P_{1} \cup S\right\}$ of singularities of $F(s)$ on the line $\sigma=\theta$. Set $\gamma=\min _{s \in P}|t|$ if $P \neq \varnothing, \gamma=+\infty$ otherwise. Then, if $\gamma \neq 0, f(x)$ changes signs on an $X$-set with a counting function $W(y)=\sum_{x_{n} \in X ; x_{0} \leqq x_{n} \leqq y} 1$ that satisfies $\lim \sup _{y \rightarrow \infty} W(y) / \log y \geqq \gamma / \pi$.

5. The method of proof. Let $a_{n}$ be a sequence of complex numbers such that the Dirichlet series $f(s)=\sum_{n=1}^{\infty} a_{n} n^{-s}$ has a finite abscissa of convergence $\sigma_{0}$. Let also $S(x)=\sum_{n<x} a_{n}$ for $x \notin Z^{+}, S(m)=\sum_{n<m} a_{n}+\frac{1}{2} a_{m}$ for $m \in Z^{+}$. In order to simplify the writing, we set $S(x)=\sum_{n}^{\prime} \leqq x a_{n}$, the prime indicating that if $x$ is integral, the last term of the sum enters with the coefficient $\frac{1}{2}$. This convention will be adhered to throughout the remainder of the paper. With this definition of $S(x)$ one has (see [31, pp. 53-54])

$$
S(x)=\frac{1}{2 \pi i} \int_{(c)} s^{-1} f(s) x^{s} d s,
$$

where the integral stands for the Cauchy value $\lim _{T \rightarrow \infty} \int_{c-i T}^{c+i T} s^{-1} f(s) x^{s} d s$; this exists at least for $c>\sigma_{0}$. By Mellin's theorem ([31, p. 33])

$$
s^{-1} f(s)=\int_{1}^{\infty} S(x) x^{-s-1} d x
$$


the integral being convergent for $\sigma \geqq \sigma_{0}$. In many cases the integral in (2) can be estimated by moving the line of integration to the left, while taking into account the residues of the integrand. In this way, $S(x)=g(x)+R(x)$ with

$$
R(x)=(2 \pi i)^{-1} \int_{\left(c^{\prime}\right)} s^{-1} f(s) x^{s} d s, \quad c^{\prime}<c
$$

and where $g(x)$ stands for the sum of the residues of the singularities of the integrand between $\sigma=c^{\prime}$ and $\sigma=c$. Let us denote $\int_{1}^{\infty} g(x) x^{-s-1} d x$ by $s^{-1} f_{1}(s)$; then we obtain from (3):

$$
F_{1}(s)=s^{-1}\left(f(s)-f_{1}(s)\right)=\int_{1}^{\infty} R(s) x^{-s-1} d x .
$$

One may now verify whether $F_{1}(s)$ satisfies the conditions of any of the theorems or Corollaries A, B, C, or D and draw the corresponding conclusion concerning $R(x)$. If Theorems $\mathrm{A}$ or $\mathrm{C}$ (or their corollaries) apply, then it follows that each of the inequalities $R(x) \gtrless 0$ holds on an $X$-set. If in addition also Theorem $\mathrm{B}$ or Theorem $\mathrm{D}$ applies, then it follows furthermore that the corresponding counting functions satisfy $W(y)=\Omega(\log y)$; occasionally one can obtain also some information concerning the constant involved in the $\Omega$-symbol.

Actually, one may do even better. Indeed, let $h(x) \geqq 0, h(x) \rightarrow+\infty$ for $x \rightarrow+\infty$ and be such that $\int_{1}^{\infty} h(x) x^{-s-1} d x$ is holomorphic for $\sigma \geqq \theta$; then, for any real constant $C$,

$$
F(s)=\int_{1}^{\infty}(R(x)-C h(x)) x^{-s-1} d x
$$

satisfies the conditions of exactly the same among the Theorems and Corollaries $\mathrm{A}, \mathrm{B}, \mathrm{C}$, or D as did $F_{1}(s)$, regardless of the size of $|C|$. It follows that, if Theorems or Corollaries $\mathrm{A}$ or $\mathrm{C}$ apply, each inequality $R(x)>C h(x)$ and $R(x)<-C h(x)$ holds on some $X$-set, for arbitrarily large (but constant) $C>0$; if also Theorems B or D applies, one gets also the corresponding refinement $W(y)=\Omega(\log y)$ for the respective $X$-sets.

6. Remarks and comments. (a) It is clear that the success of the method depends in an essential way on the existence of properly complex (i.e., nonreal) singularities of $F(s)$. One may observe that the Mellin transforms of the real valued functions $g(x)$ and $h(x)$ will not furnish these, so that the method fails, unless $f(s)=\sum_{n=1}^{\infty} a_{n} n^{-s}$ itself has such singularities. There are nontrivial situations, when this is not the case. For instance, if $r_{n}=\sum_{a^{2}+b^{2}=n} 1$, then $f(s)=\sum_{n=1}^{\infty} r_{n} n^{-s}=4 \zeta(s) L(s)$

$$
\left(L(s)=\sum_{n=0}^{\infty}(-1)^{n}(2 n+1)^{-s}\right)
$$

is holomorphic, except for the one real pole at $s=1$. However, in a great variety 
of problems, in particular in those connected with the distribution of primes, the complex singularities exist, although, sometimes, the proof of their existence is nontrivial (see $\S 11$, Theorem $E$ ). In problems involving the distribution of primes, these singularities are furnished by the zeros of the Riemann (or more general) zeta functions, or of $L$-functions.

(b) In this connection it may be worthwhile to point out the following difference between $O$-theorems and $\Omega$-theorems, as far as the role played by the Riemann (and similar) hypothesis is concerned. In the proof of theorems of the type $R(x)$ $=O(g(x))$, where $R(x)$ depends on the distribution of primes, the assumption of the $R H$ (Riemann hypothesis) permits one to obtain strong statements, with relatively simple proofs. But these statements are not theorems-at least not until the $R H$ will have been proven. On the other hand, under the assumption $\theta=1$, one is in general able to prove only more modest statements and the proofs become more difficult. But now the results are valid unconditionally, because it is usually easy to show that if $R(x)=O(g(x))$ in case $\theta=1$, then the same statement holds a fortiori if $\theta<1$.

In the case of $\Omega$-theorems, the opposite is true. It is easy (often trivial) to show that $R(x)$ has large oscillations if $\theta=1$, but such results are not theorems-at least not until it may have been shown that $\theta=1$. The proofs become more difficult and the statements more modest, when one assumes that $\theta<1$, and especially if one assumes the $R H$, i.e., that $\theta=1 / 2$. On the other hand, if under the assumption $\theta=1 / 2$ one can prove that $R(x)$ has "large" oscillations, then one can usually show quite easily that this remains a fortiori true if $\theta>1 / 2$.

7. Some classical results. In this section the method will be illustrated by a few applications that yield essentially known results, in a unified, systematic way.

(a) Let $\Lambda(n)=\log p$ if $n=p^{m}, \Lambda(n)=0$ otherwise and set $\Psi(x)=\sum_{n \leqq x}^{\prime} \Lambda(n)$; then $f(s)=\sum_{n=1}^{\infty} \Lambda(n) n^{-s}=-\zeta^{\prime}(s) / \zeta(s)$. With $R(x)=\Psi(x)-x$ and $h(x)=x^{\lambda}$, equation (4) reads

$$
\begin{aligned}
F(s) & =-\left(\zeta^{\prime}(s) / s \zeta(s)+(s-1)^{-1}+C(s-\lambda)^{-1}\right) \\
& =\int_{1}^{\infty}\left(\Psi(x)-x-C x^{\lambda}\right) x^{-s-1} d x .
\end{aligned}
$$

With $\theta$ and $P$ defined as in $\S 2$, let $\lambda<\theta$; then Theorems A and B apply and one obtains the following result:

THEOREM 1. For any pair of constants $C>0, \varepsilon>0$, the two inequalities

$$
\Psi(x)-x \gtrless \pm C x^{\theta-\varepsilon}
$$

are each verified on an $X$-set with corresponding counting function satisfying $\lim \sup _{y \rightarrow \infty} W(y) / \log y \geqq \gamma / \pi$, where $\gamma$ is the smallest ordinate of the zeros of $\zeta(s)$ of abscissa $\sigma=\theta(\gamma=+\infty$ if no such zeros exist $)$. 
REMARKS.

1. If $\theta=1$, then $\gamma=+\infty$; if $\theta=1 / 2, \gamma=14.13 \cdots$; if $1 / 2<\theta<1$, then the value of $\gamma$ is not known, but $\gamma>10,000$ (see [24]).

2. Observing that $\Psi(n+0)-n-(\Psi(n+1-0)-(n+1))=1$ and that

$$
0 \leqq \Psi(n+0)-\Psi(n-0) \leqq \log n<n^{\lambda} \quad(\lambda>0)
$$

for $n$ sufficiently large, it follows that one may chose for each inequality $X$-sets consisting of natural integers, i.e., $Z$-sets. Similar reasonings apply in many cases and will not be repeated each time the corresponding results are indicated.

3. As already stated, the particular case $C=0$ is due to Pólya [27] and was the immediate reason for proving Theorem $B$. In case $P$ is empty, the present method yields nothing better than the second member of (5). However, if $P \neq \varnothing$, then $0<\gamma<+\infty$, so that Theorem $\mathrm{C}$ and its corollary apply and we obtain

Theorem 2. If $P \neq \varnothing$, then there exists a constant $C_{0}>0$, such that for $C<C_{0}$, each of the two inequalities

$$
\Psi(x)-x \gtrless \pm C x^{\theta}
$$

holds on a Z-set.

In particular, if the $R H$ holds, then $P \neq \varnothing$, and it follows from Corollary C(iii) that $\left(5^{\prime}\right)$ holds with $\theta=1 / 2$ for every $C<\sup _{\rho=1 / 2+i t}\left|\operatorname{Res} \zeta^{\prime}(s) / s \zeta(s)\right|=\left|\rho_{1}\right|^{-1}$, where $\rho_{1}=1 / 2+i(14.13 \cdots)$, the first complex zero of $\zeta(s)$, with $\left|\rho_{1}\right| \cong 14.14 \cdots$. In case $\theta \neq 1 / 2$, Theorem 1 shows that much more is true; this illustrates the comments of $\S 6(b)$. In conclusion, remembering also the preceding Remark 2, one obtains

THEOREM $2^{\prime}$. Each of the inequalities $\Psi(x)-x \gtrless \pm(2 / 29) x^{1 / 2}$ holds on a Z-set.

This unconditional result is due essentially to E. Schmidt [29], who established it with the constant $1 / 29$. One observes that Theorems 2 and $2^{\prime}$ give no information concerning $W(y)$. While Theorem 2 cannot be improved substantially if $\theta \neq 1 / 2$ (see [2]), Theorem $2^{\prime}$ is weaker than Littlewood's [25] quoted result:

$$
\Psi(x)-x=\Omega_{ \pm}\left(x^{1 / 2} \log _{3} x\right) .
$$

One observes that Littlewood's result would follow, if Theorem C could be improved, by replacing the first member of (1) by

$$
\lim _{\sigma \rightarrow \theta^{+}} \sup \left|F^{-1}(\sigma) F(\sigma+i t) \log \log (\sigma-\theta)^{-1}\right| .
$$

This is equivalent to the

Conjecture 1. Let $T_{0}=\{t \mid \zeta(1 / 2+i t)=0\}, h(\sigma)=\log _{2}(\sigma-1 / 2)^{-1}$; then there exists a constant $C_{1}>0$, such that both functions $\Psi(x)-x \pm C_{1} x^{1 / 2}$ belong to $K_{h, T_{0}}$.

(b) Let $a_{n}=m^{-1}$ if $n=p^{m}, a_{n}=0$ otherwise and set

$$
f_{0}(x)=\sum_{p^{m} \leqq x}^{\prime} m^{-1}=\sum_{m \geqq 1} m^{-1} \pi\left(x^{1 / m}\right)
$$


then $f(s)=\sum_{n=1}^{\infty} a_{n} n^{-s}=\log \zeta(s)$ and, with $h(x)=x^{\lambda} / \log x$, (4) becomes

$$
\begin{aligned}
F(s) & =s^{-1}\left\{\log (s-1) \zeta(s)+\phi_{1}(s)-C \log (s-\lambda)^{-1}\right\} \\
& =\int_{2}^{\infty}\left\{f_{0}(x)-\operatorname{li} x-C x^{\lambda} / \log x\right\} x^{-s-1} d x,
\end{aligned}
$$

where

$$
\phi_{1}(s)=\sum_{n=1}^{\infty} \frac{(1-s)^{n} \log ^{n} 2}{n ! n}-C_{1},
$$

an entire function, and

$$
C_{1}=\int_{1}^{\infty} v^{-1} e^{-v} d v+\sum_{n=1}^{\infty} \frac{(-1)^{n}}{n ! n}-\log \log 2 .
$$

This follows by observing that, for $\sigma>1$,

$$
\begin{aligned}
\int_{2}^{\infty} \operatorname{li} x \cdot x^{-s-1} d x & =\int_{2}^{\infty} x^{-s-1}\left\{\int_{2}^{x}(\log y)^{-1} d y\right\} d x \\
& =\int_{2}^{\infty}(\log y)^{-1}\left\{\int_{y}^{\infty} x^{-s-1} d x\right\} d y \\
& =s^{-1} \int_{2}^{\infty}\left(y^{s} \log y\right)^{-1} d y
\end{aligned}
$$

and that the latter integral is equal to

$$
\begin{aligned}
\int_{\log 2}^{\infty} u^{-1} e^{-u(s-1)} d u & =\int_{(s-1) \log 2}^{\infty} v^{-1} e^{-v} d v \\
& =\int_{(s-1) \log 2}^{1} v^{-1} e^{-v} d v+\int_{1}^{\infty} v^{-1} e^{-v} d v
\end{aligned}
$$

with

$$
\begin{aligned}
\int_{(8-1) \log 2}^{1} v^{-1} e^{-v} d v & =\int_{(8-1) \log 2}^{1} v^{-1} d v+\int_{(s-1) \log 2}^{1} v^{-1}\left(e^{-v}-1\right) d v \\
& =\log \frac{1}{(s-1) \log 2}+\int_{(8-1) \log 2}^{1} v^{-1}\left\{\sum_{n=1}^{\infty}(-1)^{n} v^{n} / n !\right\} d v .
\end{aligned}
$$

For $\lambda<\theta,\left(4^{\prime}\right)$ shows that Theorems $A$ and $B$ are applicable and yield the following result:

THEOREM 3. For every couple: $C>0, \varepsilon>0$, the inequalities:

$$
f_{0}(x)-\operatorname{li} x \gtrless \pm C x^{\theta-\varepsilon}
$$

hold, each on a Z-set with corresponding counting function $W(y)=\Omega(\log y)$.

If $P=\varnothing$, the method yields no more; but if $P \neq \varnothing$, Corollary C(iv) yields:

THEOREM 4. If $P \neq \varnothing$, then the inequalities

$$
f_{0}(x)-\operatorname{li} x \gtrless \pm C x^{\theta} / \log x
$$


hold each on a Z-set, provided that $C<\sup _{\rho \in P}\left|k_{\rho} / \rho\right|, k_{\rho}$ being the multiplicity of the zero $\rho=\theta+$ it of $\zeta(s)$.

Theorems B and D are not applicable, so that nothing follows for the order of magnitude of $W(y)$.

If, in particular, the $R H$ holds, then $P \neq \varnothing$, $\sup _{\rho \in P}\left|k_{\rho} / \rho\right| \geqq\left|\rho_{1}\right|^{-1} \approx 1 / 14.14 \cdots$ and one may take in (6') $\theta=1 / 2, C=2 / 29$, say. This yields

THEOREM 4 . The inequalities $f_{0}(x)-\operatorname{li} x \gtrless \pm(2 / 29) x^{1 / 2} \log x$ hold each on $a$ Z-set.

This theorem (due with the smaller constant 1/29 to E. Schmidt), holds unconditionally, because if $\theta \neq 1 / 2$, much more follows from Theorem 3 .

As before, one may observe that if $\theta \neq 1 / 2$, then Theorem 4 cannot be improved substantially (see [2]); but, if $\theta=1 / 2$, Littlewood's result ([25]; see also [4]) $f_{0}(x)-\operatorname{li} x=\Omega_{ \pm}\left(x^{1 / 2} \log _{3} x / \log x\right)$ is stronger, does not follow from Theorems $\mathrm{C}$ or $\mathrm{C}_{1}$, but is a consequence of Conjecture 1 .

(c) If we replace $f_{0}(x)$ by $\pi(x)+\frac{1}{2} \pi\left(x^{1 / 2}\right)+O\left(x^{1 / 3}\right)=\pi(x)+x^{1 / 2} / \log x+o\left(x^{1 / 2} / \log x\right)$, it follows from (6) and $\left(6^{\prime}\right)$ that the corresponding results hold also if $f_{0}(x)$ is replaced by $\pi(x)$, provided that $\theta \neq 1 / 2$. Hence one obtains

THEOREM 5. (a) If $\theta \neq 1 / 2$, then for arbitrarily large $C>0$ and arbitrarily small $\varepsilon>0$, the inequalities $\pi(x)-\mathrm{li} x \gtrless \pm C x^{\theta-\varepsilon}$ hold each on a Z-set with corresponding counting function $W(y)=\Omega(\log y)$.

(b) If, furthermore, $P \neq \varnothing$, then each of the inequalities $\pi(x)-\operatorname{li} x \gtrless \pm C x^{\theta} / \log x$ holds on a Z-set provided that $C<\sup _{\rho \in P}\left|k_{\rho} / \rho\right|$.

If $\theta=1 / 2$ then $\left(6^{\prime}\right)$ leads to the two inequalities $\pi(x)-\operatorname{li} x>-(C+1) x^{1 / 2} / \log x$, and $\pi(x)-\operatorname{li} x<(C-1) x^{1 / 2} / \log x$, respectively, each holding on a $Z$-set. If one could take here a value for $C>1$, it would still follow that both inequalities $\pi(x)-$ li $x \gtrless 0$ hold on $X$-sets; but, as seen, the method yields only $C>2 / 29$, insufficient for this purpose. The result, of course, follows easily from Littlewood's theorem. In order to obtain it by the present method, it would be sufficient to improve (in the particular case of the present integrand) Theorem $\mathrm{C}_{1}$ to read as follows:

CONJECTURE 2. The two functions $f_{0}(x)-\operatorname{li} x \pm x^{1 / 2} / \log x$ belong to the class $K_{15, T_{0}}$.

Conjecture 2 is, of course, much weaker than Conjecture 1.

\section{The conjectures of Mertens, Pólya and Turán.}

(a) With $a_{n}=\mu(n)$, the Möbius function, and $M(x)=\sum_{n \leqq x}^{\prime} \mu(n)$, one obtains $f(s)=\sum_{n=1}^{\infty} \mu(n) n^{-s}=\zeta(s)^{-1}$ and, taking $h(x)=x^{\lambda}$, (4) specializes to

$$
F(s)=(s \zeta(s))^{-1}-C(s-\lambda)^{-1}=\int_{1}^{\infty}\left\{M(x)-C x^{\lambda}\right\} x^{-s-1} d x
$$


With $\lambda<\theta$, Theorems $\mathrm{A}$ and $\mathrm{B}$ apply and yield:

THEOREM 6. For arbitrary constants $C>0, \varepsilon>0$, the inequalities

$$
M(x) \gtrless \pm C x^{\theta-\varepsilon}
$$

hold each on Z-sets with counting functions $W(y)=\Omega(\log y)$.

The proof follows exactly the outline of $\S 5$, leading to $X$-sets; these are recognized to be actually $Z$-sets, because a change from $M(x)<C x^{\theta-\varepsilon}$ to $M(x)>C x^{\theta-\varepsilon}$ can take place only when the continuous variable $x$ takes an integral value.

If, in particular, $\theta \neq 1 / 2$, then (7) holds for some exponent $\theta-\varepsilon>1 / 2$ and arbitrarily large $C$, and one obtains

THEOREM 7. If $\theta \neq 1 / 2$, then there exists an $\varepsilon>0$, such that for arbitrarily large $C>0$ each of the inequalities

$$
M(x) \gtrless \pm C x^{1 / 2+\varepsilon}
$$

holds on a Z-set.

This (well-known) statement is inconsistent with the

Mertens Conjecture. $|M(x)| \leqq x^{1 / 2}$.

If $\theta=1 / 2$, then $P \neq \varnothing$. In case $\zeta(s)$ has a multiple zero on the critical line, Corollary C(ii) applies and leads to the following

THEOREM 7'. If $\theta=1 / 2$ and $\zeta(s)$ has a multiple zero $\rho=1 / 2+i t$, then, for arbitrary $C>0$, each of the inequalities

$$
M(x) \gtrless \pm C x^{1 / 2}
$$

holds on a Z-set.

It follows (as well known) that Mertens hypothesis implies both, the $R H$ and the simplicity of all nontrivial zeros. Even in that case the inequalities ( $\left.7^{\prime}\right)$ still hold on $Z$-sets, but our Theorems $\mathrm{C}$ and $\mathrm{C}_{1}$ permit us to infer this only for $C<\sup \left|\rho \zeta^{\prime}(\rho)\right|^{-1}$. From (7) it follows that

$$
\sup _{\rho \in P}\left|\rho \zeta^{\prime}(\rho)\right|^{-1} \geqq\left|\rho_{1} \zeta^{\prime}\left(\rho_{1}\right)\right|^{-1} \cong .0892 \cdots .
$$

One concludes that, without any unproven hypotheses, the following corollary holds:

Corollary 7. For every $C<1 / 12$, both inequalities $M(x) \gtrless \pm C x^{1 / 2}$ hold on Z-sets.

This (known, see [31, pp. 317-318]) result is not quite sufficient to disprove the Mertens conjecture. To achieve that purpose, Theorem $C_{1}$ would have to be modified (in the present, particular case) to the following version:

Conjecture 3. The functions $M(x) \pm x^{1 / 2}$ belong to the class $K_{12, T_{0}}$. 
(b) Let $a_{n}=\lambda(n)$, Liouville's function, defined for $n=p_{1} p_{2} \cdots p_{k}$ (the primes not necessarily distinct) by $\lambda(n)=(-1)^{k}$. Then $f(s)=\sum_{n=1}^{\infty} \lambda(n) n^{-s}=\zeta(2 s) / \zeta(s)(\sigma>1)$ and, setting $S(x)=\sum_{n \leqq x}^{\prime} \lambda(n)$, and $h(x)=x^{\lambda}$, (4) becomes

$$
F(s)=\zeta(2 s) / s \zeta(s)-C(s-\lambda)^{-1}=\int_{1}^{\infty}\left\{S(x)-C x^{\lambda}\right\} x^{-s-1} d x .
$$

Exactly as under (a), one obtains from (4"), using Theorems A and B the following:

THEOREM 8. For any constants $C>0, \varepsilon>0$, each of the inequalities

$$
S(x) \gtrless \pm C x^{\theta-\varepsilon}
$$

holds on a Z-set with corresponding $W(y)=\Omega(\log y)$.

In particular, one has the

COROLlaRY 8. If $\theta \neq 1 / 2$, then there exists an $\varepsilon>0$ such that, for every constant $C>0$, each of the inequalities

$$
S(x) \gtrless \pm C x^{1 / 2+\varepsilon}
$$

holds on a Z-set with corresponding $W(y)=\Omega(\log y)$.

Using Corollary C, one obtains from (4")

Theorem 9. If $\theta \neq 1 / 2$ and $P \neq \varnothing$, then there exists $C_{0}>0$, such that for $C<C_{0}$, each of the inequalities

$$
S(x) \gtrless \pm C x^{\theta}
$$

holds on a Z-set.

The restriction $\theta \neq 1 / 2$ comes, of course, from the fact that $\zeta(2 s)$ has a pole at $s=1 / 2$, the corresponding residue of $F(s)$ being $\{\zeta(1 / 2)\}^{-1}$. Hence, it follows from (4") that, for $\lambda<1 / 2$, Theorems $A$ and $B$ apply to the integrand of

$$
\frac{\zeta(2 s)}{s \zeta(s)}-\frac{1}{\zeta\left(\frac{1}{2}\right)\left(s-\frac{1}{2}\right)}-\frac{C}{s-\lambda}=\int_{1}^{\infty}\left\{S(x)-\frac{x^{1 / 2}}{\zeta\left(\frac{1}{2}\right)}-C x^{\lambda}\right\} x^{-s-1} d x ;
$$

consequently, one obtains

THEOREM 10. For every pair of constants $\varepsilon>0, C>0$, each of the inequalities

$$
S(x)-x^{1 / 2} \zeta(1 / 2)^{-1} \gtrless \pm C x^{1 / 2-\varepsilon}
$$

holds on a Z-set with $W(y)=\Omega(\log y)$.

On account of the fact that $\zeta(1 / 2) \cong-1.46 \cdots<0$, this suggests that $S(x)$ is preponderantly negative. The

CONJECTURE. $S(x) \leqq 0$ for $x \geqq 2$,

sometimes called the Pólya conjecture, does, of course, not follow-and is, in fact, 
not true, as has been shown by Haselgrove [6]. In an attempt to prove Haselgrove's result one may observe that it follows amply from Corollary 8 , if $\theta \neq 1 / 2$. If $\theta=1 / 2$, then $P \neq \varnothing$ and, taking $C=0$ in (4"), Corollary C(ii) leads to $S(x) \gtrless 0$ on $X$-sets, provided that $\zeta(s)$ has at least one multiple zero. It follows that the truth of Pólya's conjecture would have implied both, the $R H$ and the simplicity of all zeros of the Riemann zeta function. Assuming these most stringent conditions, Corollary C(iii) would still lead to $S(x) \gtrless 0$ on $X$-sets, provided that

$$
\operatorname{Res}\{F(s)\}_{s=1 / 2}<\sup _{\rho \in P}|\operatorname{Res} \zeta(2 s) / s \zeta(s)|_{s=\rho}=R_{1},
$$

say. It is clear that $R_{1} \geqq\left|\zeta\left(2 \rho_{1}\right) / \rho_{1} \zeta^{\prime}\left(\rho_{1}\right)\right| \cong .1737 \ldots$ (see [7]; the data from these tables make it almost certain that one actually has an equality here). Consequently, Theorems $\mathbf{C}$ and $\mathrm{C}_{1}$, as they stand, are insufficient for the proof of Haselgrove's result; an improvement by a factor of 4 would, however, suffice. Hence, Haselgrove's disproof of the Pólya conjecture follows from

CONJECTURE 4. $S(x) \in K_{4, T_{0}}$.

(c) Let $a_{n}=\lambda(n) / n$ and $T(x)=\sum_{n}^{\prime} \leqq x \lambda(n) / n$; then

$$
f(s)=\sum_{n=1}^{\infty}(\lambda(n) / n) n^{-s}=\zeta(2 s+2) / \zeta(s+1)
$$

and the reasonings parallel entirely those under (b). Therefore, it should be sufficient to record the results informally:

The inequalities $T(x)+x^{1 / 2} / \zeta(1 / 2) \gtrless \pm C x^{-1 / 2-\varepsilon}$ hold on $X$-sets with $W(y)$ $=\Omega(\log y)$, so that one may suspect that $T(x)$ is predominantly positive. The statement “ $T(x) \geqq 0$ for $x \geqq 1$," sometimes called the Turán conjecture, has been disproven by Haselgrove [6]. Just as before, one easily shows (using the present particular case of (4)) that each of the inequalities $T(x) \gtrless 0$ holds on an $X$-set, provided that either $\theta \neq 1 / 2$, or if $\theta=1 / 2$, that there exists a multiple zero $\rho=1 / 2+i$ of $\zeta(s)$. To draw the same conclusion in case the $R H$ holds and all zeros of $\zeta(s)$ are simple, Corollary C(iii) would require that $R_{2}=\sup _{\rho \in P}\left|\operatorname{Res}\{(2 s+2) / s \zeta(s+1)\}_{s=\rho-1}\right|$ be larger than $\left|\operatorname{Res}\{\zeta(2 s+2) / s \zeta(s+1)\}_{s=-1 / 2}\right|=|\zeta(1 / 2)|^{-1}$. From the functional equation of the Riemann zeta function it follows that, under the $R H,|\rho|=|\rho-1|$ so that $R_{2}=R_{1}$ and from here on the situation is identical with that under (b); in particular, Conjecture 4 would dispose of the Turán conjecture, while Theorems $\mathrm{C}$ and $\mathrm{C}_{1}$ are insufficient (by a factor 4) to accomplish this.

RemarK. While Pólya and Turán have drawn conclusions from the quoted "conjectures," there is no indication that either of them ever conjectured that these were actually true-and in time both statements were disproven. On the other hand, Mertens, presumably, believed that $|M(x)| \leqq \sqrt{ } x$ and this conjecture (although unlikely to hold) has not yet been disproven.

9. The function $S(x)=\sum_{n \leqq x}^{\prime} 2^{\omega(n)}$. Let $\omega(n)$ stand for the number of distinct prime factors of $n$; then $f(s)=\sum_{n=1}^{\infty} 2^{\omega(n)} n^{-s}=\zeta^{2}(s) / \zeta(2 s)(\sigma>1)$. From (2) one obtains 
$S(x)=(2 \pi i)^{-1} \int_{(c)}\left\{\zeta^{2}(s) / s \zeta(2 s)\right\} x^{s} d s(c>1)$. At $s=1$, the integrand has a pole of order 2, with residue $g(x)=6 \pi^{-2} x\left\{\log x+2 \gamma-1-2 \zeta^{\prime}(2) / \zeta(2)\right\}$, where $\gamma$ stands for the Euler-Mascheroni constant. Hence, setting $R(x)=S(x)-g(x)$ and with $h(x)=x^{\lambda}$, (4) becomes

$$
\begin{aligned}
F(s) & =\frac{\zeta^{2}(s)}{s \zeta(2 s)}-\frac{1}{\zeta(2)}\left\{\frac{1}{(s-1)^{2}}+\frac{2 \gamma-1-2 \zeta^{\prime}(2) / \zeta(2)}{s-1}\right\}-\frac{C}{s-\lambda} \\
& =\int_{1}^{\infty}\left\{R(x)-C x^{\lambda}\right\} x^{-s-1} d x .
\end{aligned}
$$

$F(s)$ is holomorphic for $\sigma>1$ and also at $s=1$, and is meromorphic in the whole (finite) complex plane, with poles at $s=0,-1,-2, \ldots$ and $s=\rho / 2$ ( $\rho$ being the zeros of $\zeta(s)$ ). Using Theorems A, B, C and their corollaries, one now obtains from $\left(4^{\prime \prime}\right)$ in a straightforward way the following theorems.

THEOREM 11. For every couple of constants $C>0, \varepsilon>0$, each of the inequalities

$$
\begin{aligned}
S(x)-g(x) & =\sum_{n \leqq x}^{\prime} 2^{\omega(n)}-6 \pi^{-2} x\left(\log x+2 \gamma-1-2 \zeta^{\prime}(2) / \zeta(2)\right) \\
& \gtrless \pm C x^{\theta / 2-\varepsilon}
\end{aligned}
$$

holds on a Z-set with $W(y)=\Omega(\log y)$.

COROLlary 11. Each of the two inequalities

$$
\sum_{n \leqq x}^{\prime} 2^{\omega(n)} \gtrless 6 \pi^{-2} x\left(\log x+2 \gamma-1-2 \zeta^{\prime}(2) / \zeta(2)\right)
$$

holds on a Z-set with corresponding counting function $W(y)=\Omega(\log y)$.

THEOREM 12. If $P \neq \varnothing$ and if there exists a multiple zero $\rho=\theta+i$, then for every constant $C>0$, each of the two inequalities

$$
S(x)-g(x) \gtrless \pm C x^{\theta / 2}
$$

holds on a Z-set. If $P \neq \varnothing$ and all zeros $\rho \neq \theta+$ it are simple, then the same result holds, provided

$$
C<C_{0}=\sup _{\rho \in P}\left|\zeta^{2}(\rho / 2) / \rho \zeta^{\prime}(\rho)\right| \cdot\left(\left|\frac{\zeta^{2}\left(\rho_{2} / 2\right)}{\rho_{2} \zeta^{\prime}\left(\rho_{2}\right)}\right| \cong .128>\left|\frac{\zeta^{2}\left(\rho_{1} / 2\right)}{\rho_{1} \zeta^{\prime}\left(\rho_{1}\right)}\right| \cong .113\right) .
$$

THEOREM 12'. If the RH holds, then for every $C<C_{0}$, both inequalities

$$
S(x)-g(x) \gtrless \pm C x^{1 / 4}
$$

hold on Z-sets.

Observing that if the $R H$ fails to hold, then Theorem 11 asserts still more, it follows that the following corollary holds unconditionally. 
COROLLARY 12. $\sum_{n \leqq x}^{\prime} 2^{\omega(n)}-6 \pi^{-2} x\left(\log x+2 \gamma-1-2 \zeta^{\prime}(2) / \zeta(2)\right)=\Omega_{ \pm}\left(x^{1 / 4}\right)$.

10. Ramanujan sums. For fixed $m \in Z^{+}$, let

$$
a_{n}=c_{n}(m)=\sum_{(h, n)=1 ; h \bmod n} \exp (2 \pi i h m / n)
$$

the Ramanujan sum, and define the function $S_{m}(x)=\sum_{n \leqq x}^{\prime} c_{n}(m)$. If one sets $\sigma_{s}(n)=\sum_{d \mid n} d^{s}$, then (see [5]) $f(s)=\sum_{n=1}^{\infty} c_{n}(m) n^{-s}=\sigma_{1-s}(n) / \zeta(s)$. Consequently, with $h(x)=x^{\lambda}$, (4) becomes

$$
F_{m}(s)=\sigma_{1-s}(m) / s \zeta(s)-C(s-\lambda)^{-1}=\int_{1}^{\infty}\left\{S_{m}(x)-C x^{\lambda}\right\} x^{-s-1} d x .
$$

Taking successively $\lambda<\theta$ and $\lambda=\theta$ and considering the alternatives $\theta>1 / 2$ and $\theta=1 / 2$, one obtains, using Theorems $\mathrm{A}, \mathrm{B}$, and $\mathrm{C}$ and Corollary $\mathrm{C}$, the following theorems.

THEOREM 13. For every copple of constants $C>0, \varepsilon>0$, each of the inequalities $S_{m}(x) \gtrless \pm C x^{\theta-\varepsilon}$ holds on a Z-set with $W(y)=\Omega(\log y)$.

THEOREM 14. If $P \neq \varnothing$ and $\rho \in P$ is a multiple zero of $\zeta(s)$, then for every constant $C>0$ each of the inequalities $S_{m}(x) \gtrless \pm C x^{\theta}$ holds on a Z-set.

TheOREM 14'. If $P \neq \varnothing$ and all $\rho \in P$ are simple zeros of $\zeta(s)$, let

$$
C_{m}=\sup _{\rho \in P}\left|\sigma_{1-\rho}(m) / \rho \zeta^{\prime}(\rho)\right| ;
$$

then, for $C<C_{m}$, each of the inequalities $S_{m}(x) \gtrless \pm C x^{\theta}$ holds on a Z-set.

Observing that if the $R H$ holds, then $P \neq \varnothing$, and if the $R H$ fails, then the result (and much more) follows from Theorem 13, it is seen that the following corollary holds unconditionally.

CoROllary 14. $S_{m}(x)=\Omega_{ \pm}\left(x^{1 / 2}\right)$.

11. Primes in distinct arithmetic progressions with the same modulus. Set $\Lambda(n, k, a)=\log p=m^{-1} \log n$ if $n=p^{m} \equiv a(\bmod k), \Lambda(n, k, a)=0$ otherwise. Also, let $k \neq 1, a, b$ be fixed natural integers satisfying $(a, k)=(b, k)=1$ and $a \neq \equiv b(\bmod k)$. Such a triplet $(k, a, b)$ of integers will be called an admissible triplet. If we set $a_{n}=\Lambda(n, k, a)-\Lambda(n, k, b)$, then

$$
S(x)=S(x ; k, a, b)=\sum_{n \leqq x}^{\prime} a_{n}=\Psi(x ; k, a)-\Psi(x ; k, b),
$$

where $\Psi(x ; k, a)=\sum_{n \leqq x}^{\prime} \Lambda(n, k, a)$ is the straightforward generalization of Tchebycheff's classical function $\Psi(x)$.

Let $\chi(n)$ be a character modulo $k$ and set $L(s, \chi)=\prod_{p}\left(1-\chi(p) p^{-s}\right)^{-1}(\sigma>1)$; 
then

$$
\begin{aligned}
\frac{L^{\prime}}{L}(s, \chi) & =-\frac{d}{d s} \sum_{p} \log \left(1-\chi(p) p^{-s}\right)=-\sum_{p} \frac{\chi(p) p^{-s}}{1-\chi(p) p^{-s}} \log p \\
& =-\sum_{p} \log p \sum_{m=1}^{\infty}\left(\chi(p) p^{-s}\right)^{m} \\
& =-\sum_{p, m} \log p \cdot \chi\left(p^{m}\right) p^{-m s}=-\sum_{n=1}^{\infty} \chi(n) \Lambda(n) n^{-s}
\end{aligned}
$$

and, denoting by $\bar{\chi}(n)$ the conjugate character,

$$
\begin{aligned}
\sum_{x \bmod k} \bar{\chi}(a) \frac{L^{\prime}}{L}(s, \chi) & =-\sum_{x \bmod k} \sum_{n=1}^{\infty} \chi(n) \bar{\chi}(a) \Lambda(n) n^{-s} \\
& =-\sum_{n=1}^{\infty} \Lambda(n) n^{-s} \sum_{x \bmod k} \chi(n) \bar{\chi}(a) \\
& =-\phi(k) \sum_{n=1: n \equiv a(\bmod k)} \Lambda(n) n^{-s} \\
& =-\phi(k) \sum_{n=1}^{\infty} \Lambda(n, k, a) n^{-s} .
\end{aligned}
$$

Consequently, the generating function of the $a_{n}$ is

$$
f(s)=f(s ; k, a, b)=\sum_{n=1}^{\infty} a_{n} n^{-s}=\phi(k)^{-1} \sum_{\chi \bmod k}(\bar{\chi}(b)-\bar{\chi}(a)) \frac{L^{\prime}}{L}(s, \chi) .
$$

The sum function $S(x)=\Psi(x ; k, a)-\Psi(x ; k, b)$ measures in some sense the difference between the number of primes $p \leqq x$ that belong to each of the two arithmetic progressions $p \equiv a(\bmod k)$ and $p \equiv b(\bmod k)$, respectively. In this sense, the present problem is one of comparative prime number theory and has recently been studied as such by Knapowski and Turán, in a sequence of papers (see [11] to [18]; also the recently appeared [19] and [20]). The present results should be compared with those obtained by them.

In the present case, $(3)$ reads

$$
\begin{aligned}
G(s) & =G(s ; k, a, b)=\frac{1}{s \phi(k)} \sum_{\chi \in K}\{\bar{\chi}(b)-\bar{\chi}(a)\} \frac{L^{\prime}}{L}(s, \chi) \\
& =\int_{1}^{\infty} S(x) x^{-s-1} d x,
\end{aligned}
$$

where it is sufficient to sum only over the set of characters $K=\{\chi \mid \chi(a) \neq \chi(b)\}$; this, in particular, eliminates the necessity of considering the principal character $\chi_{0}(n) \notin K$.

There are two main difficulties which until recently prevented the use of the present method for the problem on hand. These have been discussed in detail in 
[11, pp. 305-306]. The first one is the fact, that it is not obvious a priori that, for an arbitrary admissible triplet $(k, a, b), G(s)$ possesses the (properly) complex singularities, which are required for the success of the method, as seen in $\$ 6,(a)$. This difficulty has been overcome and the following theorem has been proven in [1]:

THEOREM E. For every admissible triplet $(k, a, b)$, the function $s G(s ; k, a, b)$ (defined by $\left.\left(3^{\prime}\right)\right)$ has a denumerable set of poles of first order $\rho=\sigma+i$, with $0 \leqq \sigma<1$ and $t \neq 0$ and nonvanishing residues

$$
m(\rho)=m(\rho ; k, a, b)=\frac{1}{\phi(k)} \sum_{\chi \in K}(\bar{\chi}(b)-\bar{\chi}(a)) m_{\rho}(\chi),
$$

where $m_{\rho}(\chi)$ stands for the multiplicity of $\rho$ as a zero of $L(s, \chi)$.

A slight modification of the proof in [1] permits one to sharpen the inequality $0 \leqq \sigma<1$ to $1 / 2 \leqq \sigma<1$. Indeed, routine computations show that the poles $\rho=i t$ contribute a function having at most bounded discontinuities. Consequently, if one denies the existence of the poles under consideration (hence, also that of the poles with $0<\sigma \leqq 1 / 2$, corresponding to the former under the functional equation), one obtains, instead of $\Psi(x ; k, a)-\Psi(x ; k, b)=g(x)+o\left(x^{-1 / 2}\right)$ (with $g(x)$ continuous) as in [1], the equation $\Psi(x ; k, a)-\Psi(x ; k, b)=g(x)+h(x)+o\left(x^{-1 / 2}\right)$, with $g(x)$, continuous and $h(x)$ having at most bounded discontinuities. It follows that, for $x=p \equiv a(\bmod k)$, the left-hand side has a discontinuity equal to $\log x$, while the right-hand side has at most a bounded discontinuity; this contradiction proves the assertion, which may be stated as

THEOREM $\mathrm{E}^{\prime}$. Theorem $\mathrm{E}$ remains valid, if one replaces the inequality $0 \leqq \sigma<1$ by $1 / 2 \leqq \sigma<1$.

Let $\theta_{k}=\theta_{k}(a, b)=\sup \left\{\sigma \mid G(\sigma+i t)^{-1}=0, t \neq 0\right\}$ and set, as before,

$$
P=\left\{\rho=\theta_{k}+i t \mid G(\rho)^{-1}=0, t \neq 0\right\}
$$

the possibility $P=\varnothing$ is not ruled out. Let also $\Sigma=\left\{\sigma_{1}, \sigma_{2}, \ldots, \sigma_{r}\right\}$ stand for the (possibly empty) set of poles of $G(s)$ on the real axis and with $\theta_{k} \leqq \sigma_{j}<1$. The second of the difficulties mentioned in [11] is the possibility that $\Sigma \neq \varnothing$. In order to avoid it, in [11] to [18] the consideration is often restricted to those integers $k$, for which the following condition holds:

Condition $\mathrm{H}$ (OF Haselgrove). An integer $k$ is said to verify Condition $\mathrm{H}$, if there exists a positive $A=A(k)$ such that $L(s, \chi) \neq 0$ for $0<\sigma<1,|t| \leqq A(k)$ and all $\chi \bmod k$.

In what follows we shall find that the following condition (formally weaker, but, presumably, equally difficult to verify) will be sufficient for our purpose.

Condition $\mathrm{H}_{1}$. An admissible triplet $(k, a, b)$ is said to satisfy Condition $\mathrm{H}_{1}$, or to 
belong to the class $H_{1}$ (written $\left.(k, a, b) \in H_{1}\right)$ if and only if the corresponding set $\Sigma$ is empty.

If $c_{j}$ are the residues of the poles $\sigma_{j} \in \Sigma$ and $C$ is an arbitrary real number, then from ( $\left.3^{\prime}\right)$ immediately follows

$$
\begin{aligned}
F(s) & =G(s)-\sum_{j=1}^{r} c_{j}\left(s-\sigma_{j}\right)^{-1}-C(s-\lambda)^{-1} \\
& =\int_{1}^{\infty}\left\{S(x)-\sum_{j=1}^{r} c_{j} x^{\sigma_{j}}-C x^{\lambda}\right\} x^{-s-1} d x .
\end{aligned}
$$

Let $\lambda<\theta\left(=\theta_{k}\right)$; then it follows from the definitions of $\Sigma$ and $c_{j}$ that $F(s)$ is holomorphic for $\sigma>\theta$ (where $\theta \geqq 1 / 2$, by Theorem $E^{\prime}$ ), but not in any half-plane $\sigma>\theta-\varepsilon(\varepsilon>0)$ and also holomorphic at $s=\theta$. Theorems A and B are, therefore, applicable. Here, as above, in listing the results we shall suppress, for simplicity, any mention of the integer $k$, whenever this can be done without danger of confusion.

THEOREM 15. For every couple of constants $C>0, \varepsilon>0$, each of the inequalities

$$
\Psi(x ; a)-\Psi(x ; b)-\sum_{j=1}^{r} c_{j} x^{\sigma_{j}} \gtrless \pm C x^{\theta-\varepsilon}
$$

holds on a Z-set with counting function $W(y)=\Omega(\log y)$.

If $\Sigma \neq \varnothing$, let $\sigma_{M}=\max _{\sigma_{j} \in \Sigma} \sigma_{j}$; then $\lim _{x \rightarrow \infty} x^{-\sigma_{M}}\{\Psi(x ; a)-\Psi(x ; b)\}=c_{M}$ so that the difference $\Psi(x ; a)-\Psi(x ; b)=c_{M} x^{\sigma_{M}}(1+o(1))$ keeps a constant sign for $x$ sufficiently large, as indicated already in [11, p. 313]. In the particular case $(k, a, b) \in H_{1}$ one obtains

CoROllary 15. If $(k, a, b) \in H_{1}$, then, for every couple of constants $C>0, \varepsilon>0$, each of the inequalities

$$
\Psi(x ; a)-\Psi(x ; b) \gtrless \pm C x^{\theta-\varepsilon}
$$

holds on a Z-set with counting function $W(y)=\Omega(\log y)$.

If for an admissible triplet one also has $P \neq \varnothing$ (and, by Theorem $\mathrm{E}^{\prime}$, this is certainly the case if $\theta_{k}=1 / 2$ ), one obtains

THEOREM 16. If $(k, a, b) \in H_{1}$ and $P \neq \varnothing$, then, for every constant

$$
C<\sup _{\rho \in P}|m(\rho) / \rho|,
$$

each of the inequalities

$$
\Psi(x ; a)-\Psi(x ; b) \gtrless \pm C x^{\theta}
$$

holds on a Z-set. 
Observing that for $\theta \neq 1 / 2$ Corollary 15 asserts much more, it follows that the next corollary holds without any restriction on $\theta$ :

Corollary 16. If $(k, a, b) \in H_{1}$ and $C<C_{0}$ (computable constant), each of the inequalities

$$
\Psi(x ; a)-\Psi(x ; b) \gtrless \pm C x^{1 / 2}
$$

holds on a Z-set.

Similarly, without any restriction on $\theta\left(=\theta_{k}\right)$, one obtains from (8)

THEOREM 17. If $(k, a, b) \in H_{1}$, then for every couple of constants $C>0, \varepsilon>0$, each of the inequalities

$$
\Psi(x ; a)-\Psi(x ; b) \gtrless \pm C x^{1 / 2-\varepsilon}
$$

holds on a Z-set with counting function $W(y)=\Omega(\log y)$.

While there is some difference in the formulation, these results should be compared with those of [12] and [13, pp. 315-317] (valid only for $a \equiv 1(\bmod k)$ and with $W(y)=\Omega\left(\log _{4} y\right)$ ); those of [14, p. 32] (referring to $k=8$ and with $W(y)=\Omega(\log \log y))$, those of [15, pp. 44-45] (which assume a weak form of the generalized Riemann hypothesis and yield $W(y)=\Omega(\log \log y))$; also with [17, p. 242] (where the infinity of sign changes is proven assuming $H$, with some (very weak) estimate for $W(y))$.

12. The function $f_{0}(x ; k, a)$. Let $g_{k}(n, a)=\Lambda(n, k, a) / \log n$ and as in $\S 3$ set

$$
f_{0}(x, k, a)=\sum_{n \leqq x}^{\prime} g_{k}(n, a)=\sum_{p^{m} \leqq x}^{\prime} m^{-1}=\sum_{m \geqq 1}^{\prime} m^{-1} \sum_{p \leqq x^{1 / m} ; p^{m} \equiv a(\bmod k)}^{k} 1 .
$$

Generalizing the classical notation $\pi(y ; k, a)=\sum_{p \leqq y: p \equiv a(\bmod k)}^{\prime} 1$, one may define $\pi\left(y ; k, a^{1 / m}\right)=\sum_{p \leqq y: p^{m}}^{\prime} \equiv a(\bmod k) 1$; then one obtains

$$
f_{0}(x ; k, a)=\sum_{m \geqq 1} m^{-1} \pi\left(x^{1 / m} ; k, a^{1 / m}\right) .
$$

The sum is finite, because $\pi\left(x^{1 / m} ; k, a^{1 / m}\right)>0$ only for $m \leqq \log x / \log 2$. As before, the integer $k$ being kept fixed, its mention as subscript, or argument will be suppressed, whenever this is possible without danger of confusion. Also, any congruence without indication of the modulus is to be understood modulo $k$.

For future reference it is convenient to recall a well-known lemma. Let $n(k, a)$ be the number of solutions of the congruence $x^{2} \equiv a(\bmod k)$, distinct $\bmod k$, and write $n(k)$ for $n(k, 1)$. For $(a, k)=1$, define the symbol of quadratic residuacy $\delta(a)$ by $\delta(a)=1$ if $n(k, a)>0, \delta(a)=0$ otherwise. If $k=p$, a prime, then it is clear that $\delta(a)=\frac{1}{2}(1+(a / p))$ and $n(k, a)=2 \delta(a)$, but this is not true in general (counterexamples: $k=8, n(8)=4, \delta(1)=1, \delta(a)=0$ for odd $a \not \equiv 1(\bmod 8)$; or, less trivially, 
$k=28, n(28)=4$, with $\delta(a)=1$ for $a=1^{2}, 3^{2}, 5^{2}$ only). However the following lemma holds.

LEMMA. For every integer $a$ with $(k, a)=1, n(k, a)=n(k) \delta(a)$.

The statement is trivial if $a$ is a nonresidue, when $n(k, a)=\delta(a)=0$ and, if $a$ is a quadratic residue $\bmod k$, the proof reduces to a verification that $n(k, a)$ is the same for all quadratic residues $a$.

Returning now to $f_{0}(x, a)$, it follows from (9) that

$$
f_{0}(x, a)=\pi(x, a)+\frac{1}{2} \sum_{c^{2} \equiv a} \pi\left(x^{1 / 2}, c\right)+\sum_{m \geqq 3} m^{-1} \pi\left(x^{1 / m}, a^{1 / m}\right) .
$$

The last term satisfies

$$
\begin{aligned}
0 & \leqq \sum_{3 \leqq m \leqq \log x / \log 2} m^{-1} \pi\left(x^{1 / 3}\right) \leqq \pi\left(x^{1 / 3}\right) \log (\log x / \log 2) \\
& =O\left(x^{1 / 3}(\log x)^{-1} \log \log x\right)=o\left(x^{1 / 3}\right) .
\end{aligned}
$$

Next, by the Walfish-Paige theorem (see, e.g., [28, p. 138]), if $(c, k)=1$, then

$$
\pi\left(x^{1 / 2}, c\right)=\pi\left(x^{1 / 2}\right) / \phi(k)+O\left(x^{1 / 2} \exp \left(-\alpha \log ^{1 / 2} x\right)\right) .
$$

Consequently,

$$
f_{0}(x, a)=\pi(x, a)+\frac{1}{2} \frac{1}{\phi(k)} \pi\left(x^{1 / 2}\right) \sum_{c^{2} \equiv a} 1+O\left(x^{1 / 2} \exp \left(-\alpha \log ^{1 / 2} x\right)\right)
$$

and, finally, using the preceding lemma,

$$
f_{0}(x, a)=\pi(x, a)+\delta(a) n(k) \pi\left(x^{1 / 2}\right) / 2 \phi(k)+o\left(x^{1 / 2} \log ^{-m} x\right)
$$

for every fixed $m$.

13. The oscillations of $f_{0}(x, a)-f_{0}(x, b)$. Let $(k, a, b)$ be an admissible triplet and, with the notations of $\S 12$, set $a_{n}=a(n ; k, a, b)=g(n, a)-g(n, b)$. Then $S_{1}(x)=\sum_{n \leqq x}^{\prime} a_{n}=f(x, a)-f(x, b)$. Proceeding essentially as in $\S 11$, one obtains instead of $\left(3^{\prime}\right)$,

$$
\frac{1}{\phi(k) s} \sum_{\chi \in K}(\bar{\chi}(a)-\bar{\chi}(b)) \log L(s, \chi)=\int_{1}^{\infty}\left\{f_{0}(x, a)-f_{0}(x, b)\right\} x^{-s-1} d x .
$$

For $x<2, f(x, a)=f(x, b)=0$; hence, the lower limit of the integral may be replaced by 2 . With the same definition for $c_{j}, \sigma_{j}(1 \leqq j \leqq r), \Sigma$ and $\theta\left(=\theta_{k}(a, b)\right)$ as in $\$ 11$, one obtains from ( $\left.3^{\prime \prime}\right)$

$$
\begin{aligned}
F_{1}(s) & =\frac{1}{\phi(k) s} \sum_{x \in K}\{\bar{\chi}(a)-\bar{\chi}(b)\} \log L(s, \chi)-\sum_{j=1}^{r} c_{j} \log \frac{1}{s-\sigma_{j}}-C \log \frac{1}{s-\lambda}+\phi(s) \\
& =\int_{2}^{\infty}\left\{f(x, a)-f(x, b)-\sum_{j=1}^{r} c_{j} x^{\sigma_{j}} / \log x-C x^{\lambda} / \log x\right\} x^{-s-1} d x
\end{aligned}
$$


here $\phi(s)$ is an entire function and the proof of $\left(8^{\prime}\right)$ follows so closely the corresponding one of $\S 7\left(\operatorname{after}\left(4^{\prime}\right)\right)$, that a repetition is unnecessary. If $\lambda<\theta$, then it follows from the definitions of $c_{j}$ and $\sigma_{j}$, that $F_{1}(s)$ is holomorphic in the halfplane $\sigma>\theta$, and also at $s=\theta$, but in no half-plane $\sigma>\theta-\varepsilon(\varepsilon>0)$. Theorems A, B or $\mathrm{D}$ and $\mathrm{E}^{\prime}$ are applicable and one obtains the following result.

THEOREM 18. Let $(k, a, b)$ be an admissible triplet; then, for arbitrarily small $\varepsilon>0$ and arbitrarily large $C>0$, both inequalities

$$
f_{0}(x, a)-f_{0}(x, b)-\sum_{j=1}^{r} c_{j} x^{\sigma_{j}} / \log x \gtrless \pm C x^{\theta-\varepsilon}
$$

hold, each on a Z-set with counting function $W(y)=\Omega(\log y)$.

If the set $P=\left\{\rho=\theta+i t \mid F_{1}(s)\right.$ singular at $\left.s=\rho, t \neq 0\right\}$ is not empty (and this is certainly the case if $\left.\theta=\theta_{k}(a, b)=1 / 2\right)$, then Corollary C(iv) applies and one obtains

THEOREM 19. If $(k, a, b)$ is an admissible triplet with $P \neq \varnothing$, then there exists $a$ (computable) constant $C_{0}>0$, such that for $C<C_{0}$, each of the two inequalities

$$
f_{0}(x, a)-f_{0}(x, b)-\sum_{j=1}^{r} c_{j} x^{\sigma_{j}} / \log x \gtrless \pm C x^{\theta} / \log x
$$

holds on a Z-set.

In case $\Sigma \neq \varnothing$ and $\sigma_{M}=\max \left\{\sigma_{j} \mid \sigma_{j} \in \Sigma\right\}$, one infers the

Corollary 19. If $(k, a, b)$ is an admissible triplet with $P \neq \varnothing$ and $\Sigma \neq \varnothing$, then

$$
f_{0}(x, a)-f_{0}(x, b)=C_{M} x^{\sigma_{M}}(\log x)^{-1}(1+o(1)) .
$$

In particular, the difference $f_{0}(x, a)-f_{0}(x, b)$ is ultimately of a constant sign.

If, on the other hand $(k, a, b) \in H_{1}$, one obtains by Theorems $\mathrm{A}, \mathrm{B}$, or $\mathrm{D}$, Corollary $\mathrm{C}(\mathrm{iv})$ and Theorems $\mathrm{E}^{\prime}$ the following results.

THEOREM 20. If $(k, a, b)$ is an admissible triplet and $(k, a, b) \in H_{1}$, then, for every $\varepsilon>0$ and $C>0$, each of the two inequalities

$$
f_{0}(x, a)-f_{0}(x, b) \gtrless \pm C x^{\theta-\varepsilon}
$$

is satisfied on a Z-set with counting function $W(y)=\Omega(\log y)$.

TheOREM 21. If $(k, a, b)$ is admissible, $(k, a, b) \in H_{1}$ and $P \neq \varnothing$, then there exists a (computable) constant $C_{0}$, such that for $C<C_{0}$, each of the two inequalities

$$
f_{0}(x, a)-f_{0}(x, b) \gtrless \pm C x^{\theta} / \log x
$$

holds on a Z-set.

If $\theta=1 / 2$, then, by Theorem $\mathrm{E}^{\prime}, P \neq \varnothing$ and one obtains 
COROLlaRy 21. For all admissible triplets $(k, a, b) \in H_{1}$, there exists a (computable) constant $C_{0}$, such that for $C<C_{0}$, both inequalities

$$
f_{0}(x, a)-f_{0}(x, b) \gtrless \pm C x^{1 / 2} / \log x
$$

hold, each on a Z-set.

Corollary 21 , obtained on the assumption $\theta=1 / 2$, holds without this restriction, because, if $\theta>1 / 2$, more follows from Theorem 20 .

While not of exactly the same nature (and with the notation $\Pi(x ; k, a)$ for our function $f_{0}(x, a)$ ), the results of this section should be compared with those in [12, pp. 316-318] (valid under the assumption $H$ and only for $a \equiv 1(\bmod k)$, and with $W=\Omega(\log \log y)$-but guaranteeing an upper bound for the first sign change); in [14, p. 32] (valid for $k=8$ only, with $W(y)=\Omega(\log \log y)$ ); and in [15, p. 44] (valid assuming the "finite form" of the extended Riemann hypothesis and with $W(y)=\Omega(\log \log y)$, for the second member in Theorem 20 or Corollary 21 of this section replaced by $\left.x^{1 / 2} \exp \left(-C \log x \log _{3} x / \log _{2} x\right)\right)$.

14. The oscillations of $\pi(x ; k, a)-\pi(x ; k, b)$. From (10) follows $\pi(x, a)-\pi(x, b)=f_{0}(x, a)-f_{0}(x, b)-(\delta(a)-\delta(b)) n(k) \pi\left(x^{1 / 2}\right) / 2 \phi(k)+o\left(x^{1 / 2} \log ^{-m} x\right)$.

In particular, if $\delta(a)=\delta(b)$ (i.e., if $a$ and $b$ have the same quadratic character $\bmod k$, by being either both quadratic residues, or both quadratic nonresidues), then

$$
\pi(x, a)-\pi(x, b)=f_{0}(x, a)-f_{0}(x, b)+o\left(x^{1 / 2} \log ^{-m} x\right) .
$$

Consequently, one obtains

Theorem 22. If $\delta(a)=\delta(b)$, then Theorems 18, 19, 20, 21 and Corollary 21 hold if we replace the function $f_{0}(x, a)-f_{0}(x, b)$ by $\pi(x, a)-\pi(x, b)$.

It should not be necessary to restate all these theorems for $\pi(x, a)-\pi(x, b)$; therefore, we indicate only the corollary, which holds unconditionally:

Corollary $21^{\prime}$. For every admissible triplet $(k, a, b) \in H_{1}$, with $\delta(a)=\delta(b)$, there exists a computable constant $C_{0}>0$, such that for $C<C_{0}$, each of the two inequalities

$$
\pi(x, a)-\pi(x, b) \gtrless \pm C x^{1 / 2} / \log x
$$

holds on a Z-set.

The problem is more difficult if $\delta(a) \neq \delta(b)$. Without loss of generality, let $\delta(a)=0$, $\delta(b)=1$. From $(10)$ now follows

$$
\pi(x, a)-\left\{\pi(x, b)+\frac{1}{2}(n(k) / \phi(k)) \pi\left(x^{1 / 2}\right)\right\}=f_{0}(x, a)-f_{0}(x, b)+o\left(x^{1 / 2} \log ^{-m} x\right) .
$$

If an admissible triplet $(k, a, b)$ has $\Sigma=\varnothing$ (as is certainly the case, e.g., for $k=4$ ), 
so that (see Theorem 20) $f_{0}(x, a)-f_{0}(x, b)$ oscillates more or less symmetrically around zero, then (11) shows in what quite precise sense there are more primes in a progression of quadratic nonresidues, than in one of quadratic residues, bearing out Tchebycheff's assertion concerning primes $p \equiv 3(\bmod 4)$ and $p \equiv 1(\bmod 4)$, respectively.

In case $\Sigma \neq \varnothing$, then the new term $\frac{1}{2}(n(k) / \phi(k)) \pi\left(x^{1 / 2}\right) \cong(n(k) / \phi(k)) x^{1 / 2} \log ^{-1} x$ is negligible with respect to the terms $x^{\sigma} / \log x(\sigma \in \Sigma)$ and (for $\varepsilon>0$ sufficiently small) to the second member in Theorems 18, 19, 20, and 21, for $\theta\left(=\theta_{k}(a, b)\right)>1 / 2$. Consequently, if $\theta \neq 1 / 2$, these theorems also remain valid with $\pi(x, a)-\pi(x, b)$ instead of $f_{0}(x, a)-f_{0}(x, b)$, and we obtain:

THEOREM 23. If $(k, a, b)$ is an admissible triplet, $(k, a, b) \notin H_{1}$, then

$$
\pi(x, a)-\pi(x, b)=c_{M} x^{\sigma_{M}}(1+o(1))
$$

where $\sigma_{M}=\max \{\sigma \mid \sigma \in \Sigma\}$; in particular, this difference is ultimately of constant sign.

THeOREM 24. If $(k, a, b)$ is an admissible triplet with $\theta=\theta_{k}(a, b) \neq 1 / 2$, then for every $C$ and sufficiently small $\varepsilon>0$, each of the inequalities

$$
\pi(x, a)-\pi(x, b)-\sum_{j=1}^{r} c_{j} x^{\sigma}, / \log x \gtrless \pm C x^{\theta-\varepsilon}
$$

holds on a Z-set, with corresponding counting function $W(y)=\Omega(\log y)$.

COROLlary 24. If the triplet $(k, a, b)$ satisfies the conditions of Theorem 24 and $(k, a, b) \in H_{1}$, then, with $C$ and $\varepsilon$ as in Theorem 24 , each of the inequalities

$$
\pi(x, a)-\pi(x, b) \gtrless \pm C x^{\theta-\varepsilon}
$$

holds on a Z-set with corresponding counting function $W(y)=\Omega(\log y)$.

TheOREM 25. If $(k, a, b)$ is admissible, with $\theta>1 / 2$ and $P \neq \varnothing$, then there exists a (computable) constant $C_{0}>0$, such that, for $C<C_{0}$, each of the inequalities

$$
\pi(x, a)-\pi(x, b)-\sum_{j=1}^{r} c_{j} x^{\sigma_{j}} / \log x \gtrless \pm C x^{\theta} / \log x
$$

holds on a Z-set.

COROllary 25. If $(k, a, b) \in H_{1}$ and satisfies the conditions of Theorem 25 , then, for $C<C_{0}$, each of the inequalities

$$
\pi(x, a)-\pi(x, b) \gtrless \pm C x^{\theta} / \log x
$$

holds on a Z-set.

It remains to consider only the case $(k, a, b) \in H_{1}, \delta(a)=0, \delta(b)=1, \theta=\theta_{k}(a, b)$ $=1 / 2$. Then $\left(8^{\prime}\right)$, Corollary 21 and (11) yield the following result: 
THEOREM 26. Let $(k, a, b)$ be an admissible triplet $(k, a, b) \in H_{1}$ and, such that $\delta(a)=0, \delta(b)=1, \theta=1 / 2$ and set

$$
C_{0}=\sup _{\rho=1 / 2+i t \in P}\left|\left\{\operatorname{Res} F_{1}(s)\right\}_{s=\rho}\right|=\sup _{\rho \in P}\left|\frac{m(\rho)}{\rho}\right| .
$$

Then, for $C<C_{0}$, each of the two inequalities

and

$$
\pi(x, a)-\pi(x, b)>\left(C+\frac{n(k)}{\phi(k)}\right) \frac{x^{1 / 2}}{\log x}
$$

holds on a Z-set.

$$
\pi(x, a)-\pi(x, b)<-\left(C-\frac{n(k)}{\phi(k)}\right) \frac{x^{1 / 2}}{\log x}
$$

COROLlaRY 26. Let $k$ be an integer with the property that for all admissible triplets, $(k, a, b) \in H_{1}$, and furthermore, that for all admissible triplets satisfying the conditions of Theorem 26 , one has $C_{0}>n(k) / \phi(k)$; then, for all admissible triplets $(k, a, b)$, each of the two inequalities

$$
\pi(x, a)-\pi(x, b) \gtrless 0
$$

holds on a Z-set.

The conditions of Corollary 26 are not always satisfied. For instance, if $k=4$, $a=3, b=1$, then $L(s, \chi)$ has as first nontrivial zero $\rho_{1}=\frac{1}{2}+6.0209 \cdots i$ (here $\chi$ is the only nonprincipal character mod 4 and the value of $\rho_{1}$ is due to Haselgrove; see [18, p. 254]). Using $\rho_{1}$ (and the known zeros of $L\left(s, \chi_{0}\right)$ on $\sigma=1 / 2$, which are the same as those of $\zeta(s)$ ) it follows only that $C_{0} \geqq\left|\rho_{1}\right|^{-1} \cong 1 / 6.04$. It is fairly certain that $C_{0}$ is actually equal to this value, because, as far as the computations go, $m_{\rho}(\chi)=1$ (or 0 ), $\phi(4)=2$ and, for $\chi \in K,|\bar{\chi}(1)-\bar{\chi}(3)|=2$, while $\left|\rho_{v}\right| \rightarrow+\infty$. On the other hand, $n(4) / \phi(4)=>1 / 6.04$. Consequently, the

CONJECTURE. For all admissible triplets $(k, a, b)$, the difference $\pi(x, a)-\pi(x, b)$ changes signs on an $X$-set,

does not follow from Corollary 26 and, indeed, cannot be inferred from the present results. The particular case $k=4$ has been proven by Hardy and Littlewood (see [4, p. 127]); as already mentioned in $\S 1$, the detailed proof does not seem to have been published by Hardy and Littlewood, but the corresponding result easily follows from [12, Theorem 5.4], by taking there $k=4$. The same holds for $k=3$ and $k=6$, for which $\phi(k)=2$. The case $k=8$ follows combining Theorem 5.4 in [12] with Theorem 1.1 in [14]. As far as I know, for no other modulus $k$ does there exist a complete proof of this "conjecture" (for $k=5$, the cases $a=2,3, b=1$ are settled by Theorem 5.1 of [12], the cases $a=1, b=4$ and $a=2, b=3$ follow from Corollary 21 and Theorem 22, and are also covered-the first case explicitly, the second implicitly-by Theorems 2.1 and 2.2 in [13]; but the cases $a=2$, or 3 with $b=4$ are, apparently, still open). 
The method of Hardy and Littlewood [4], is, presumably, applicable to any specific $k$, but could hardly be expected to give the general result for arbitrary $k$, because it depends in an essential way on the numerical values of the zeros of $L(s, \chi)$, for all $\chi(\bmod k)$. The result would follow, however, immediately, if one could infer from $\left(8^{\prime}\right)$ and $P \neq \varnothing$, that Theorem 19 holds with the second member replaced by $\pm C(x) x^{\theta} / \log x$, with $\lim _{x \rightarrow \infty} C(x)=+\infty$. In its turn, this last result would be an immediate consequence of the

CONJECTURE 5. Let $T=\left\{t_{n}\right\}$, be any set with $0<t_{1}<t_{2}<\cdots<t_{n}<\cdots$, and with counting function $W(y)=\Omega(y \log y)$; then there exists a function $C(x)$, monotonically increasing, with $\lim _{x \rightarrow \infty} C(x)=+\infty$ and with the following property: Let $h(\sigma)=\int_{2}^{\infty} C(x)(\log x)^{-1} x^{-\gamma-1 / 2} d x$; then, for every admissible triplet $(k, a, b)$, the functions $f_{0}(x, a)-f_{0}(x, b) \pm C(x) x^{1 / 2} / \log x$ belong to the class $K_{h, T}$.

15. Some particular cases. In view of the situation described in the preceding section, the following special results seem to be of interest. Let $P_{1} \subseteq P$ be the set of $\rho$ 's that are zeros of exactly one function $L(s, \chi)$. (All available evidence suggests that actually $P_{1}=P$, but no proof exists.) Then

$$
\begin{aligned}
C_{0} & \geqq \sup _{\rho \in P_{1}}\left|\frac{m(\rho)}{\rho}\right|=\sup _{\rho \in P_{1}}\left|\frac{1}{\rho} \frac{1}{\phi(k)}\left(\bar{\chi}_{j(\rho)}(a)-\bar{\chi}_{j(\rho)}(b)\right) m_{\rho}\left(\chi_{j(\rho)}\right)\right| \\
& \geqq \sup _{\rho \in P_{1}}\left\{\frac{1}{\rho} \frac{1}{\phi(k)}\left|\bar{\chi}_{j(\rho)}(a)-\bar{\chi}_{j(\rho)}(b)\right|\right\} \\
& \geqq \frac{1}{\phi(k)} \frac{1}{\left|\rho_{1}\right|} \min _{\chi_{j} \in K}\left|\bar{\chi}_{j}(a)-\bar{\chi}_{j}(b)\right|
\end{aligned}
$$

with $\left|\rho_{1}\right|=\min _{\rho \in P_{1}}\left\{|\rho| \mid L\left(\rho, \chi_{j}\right)=0\right\}$. If $\chi=\chi_{j\left(\rho_{1}\right)}$ is a real character, the last factor equals 2; otherwise, it follows from $\chi \in K$ that $\min |\bar{\chi}(a)-\bar{\chi}(b)| \geqq 2 \sin (\pi / \phi(k))$. The condition $C_{0}>n(k) / \phi(k)$ of Corollary 26 will, therefore, hold, provided that there exists, for a real, nonprincipal character $\chi$, a zero $\rho_{1}$ of $L(s, \chi)$, not shared by any $L\left(s, \chi^{\prime}\right)\left(\chi^{\prime} \neq \chi\right)$, and such that $2 /\left|\rho_{1}\right|>n(k)$, or

$$
\left|\rho_{1}\right|<2 / n(k) \text {. }
$$

For a complex character $\chi$, it is necessary that there exists a zero $\rho_{1}$ of $L(s, \chi)$ not shared by any $L\left(s, \chi^{\prime}\right)\left(\chi^{\prime} \neq \chi\right)$ and such that $2 \sin (\pi / \phi(k)) /\left|\rho_{1}\right|>n(k)$, or

$$
\left|\rho_{1}\right|<(2 / n(k)) \sin (\pi / \phi(k)) \text {. }
$$

These results are formalized in the following

THEOREM 27. Let $k$ be such that for all admissible triplets, $(k, a, b) \in H_{1}$; if there exists a character $\chi(\bmod k)$ and a complex number $\rho$ such that $L(\rho, \chi)=0, L\left(\rho, \chi^{\prime}\right) \neq 0$ for $\chi^{\prime} \neq \chi$ and such that also (12), respectively (12') holds, then, for all admissible triplets $(k, a, b)$, the difference $\pi(x, a)-\pi(x, b)$ changes signs on a Z-set. 
Proof. If $\theta_{k}(a, b)>1 / 2$, the result (and more) follows from Corollary 24; one may, therefore, assume $\theta_{k}(a, b)=1 / 2$, so that either Theorem 22 or Corollary 26 apply and the result follows from $C_{0}>n(k) / \phi(k)$.

COROLlARY 27. If the prime $p$ is such that for all admissible triplets, $(p, a, b) \in H_{1}$, then $\pi(x ; p, a)-\pi(x ; p, b)$ changes signs on a Z-set, provided that there exists $a$ real character $\chi(\bmod k)$ and $a \rho=\beta+i \gamma$, such that $L(\rho, \chi)=0, L\left(\rho, \chi^{\prime}\right) \neq 0\left(\chi^{\prime} \neq \chi\right)$, with $|\rho|<1$, i.e., with $|\gamma|<\sqrt{ } 3 / 2$.

Proof. Follows from Theorem 27, observing that $n(p)=2$ and $\phi(p)=p-1$.

THEOREM 28. The difference $\pi(x ; k, a)-\pi(x ; k, b)$ changes signs infinitely often, for all admissible triplets corresponding to the moduli $k=3,4,6,8,43,67,163$.

Proof. The case $k=4$ has been settled by Hardy and Littlewood [4], and $k=3,6$ and 8 by Knapowski and Turán [14]. For $k=43,67$ and 163, the result follows from the existence of (not shared) zeros of the corresponding unique $L(s, \chi)$ with real nonprincipal character at $\rho=1 / 2+i$ with $t=.8364 \cdots, t=.6043 \cdots$ and $t=.2029 \cdots$, respectively (see $[0$, p. 129]) all leading to $|\rho|<1$.

\section{BIBLIOGRAPHY}

0. D. Davies and C. B. Haselgrove, Proc. Roy. Soc. Ser. A 264 (1961), 122-132.

1. E. Grosswald, C. R. Acad. Sci. Paris 260 (1965), 4299-4302.

2. —, C. R. Acad. Sci. Paris 260 (1965), 3813-3816.

3. - Israel J. Math. 3 (1965), 211-220.

4. G. H. Hardy and J. E. Littlewood, Acta Math. 41 (1918), 119-196.

5. G. H. Hardy and E. M. Wright, An introduction to the theory of numbers, $3 \mathrm{rd}$ ed., The Clarendon Press, Oxford, 1954.

6. C. B. Haselgrove, Mathematika 5 (1958), 141-145.

7. C. B. Haselgrove and J. C. P. Miller, Tables of the Riemann zeta function, Royal Soc. Math. Tables, Vol. 6, Cambridge Univ. Press, Cambridge, 1960.

8. A. E. Ingham, Acta Arith. 1 (1936), 201-211.

9. - The distribution of prime numbers, Cambridge Univ. Press, Cambridge, 1932.

10. J. L. W. V. Jensen, Acta Math. 22 (1899), 359-364.

11. S. Knapowski and P. Turán, Acta Math. Acad. Sci. Hungar. 13 (1962), 229-314.

12. - Acta Math. Acad. Sci. Hungar. 13 (1962), 315-342.

13. - Acta Math. Acad. Sci. Hungar. 13 (1962), 343-364.

14. —, Acta Math. Acad. Sci. Hungar. 14 (1963), 31-42.

15. —, Acta Math. Acad. Sci. Hungar. 14 (1963), 43-63.

16. —- Acta Math. Acad. Sci. Hungar. 14 (1963), 65-78.

17. —- Acta Math. Acad. Sci. Hungar. 14 (1963), 241-250.

18. — Acta Math. Acad. Sci. Hungar. 14 (1963), 251-268.

19. —- Acta Arith. 9 (1964), 23-40.

20. —, J. Analyse Math. 14 (1965), 267-274.

21. H. von Koch, Acta Math. 24 (1901), 159-182.

22. ——, Math. Ann. 55 (1902), 441-464.

23. E. Landau, Math. Ann. 59 (1905), 527-550.

24. D. H. Lehmer, Mathematika 3 (1956), 102-108. 
25. J. E. Littlewood, C. R. Acad. Sci. Paris 158 (1914), 1862-1872.

26. E. Phragmén, Öfversigt of Kong. Vetensk.-Akad. Förhandlingar, Stockholm, Vol. 48, 1898; pp. 559-616.

27. G. Pólya, Nachr. Ges. Wiss. Göttingen (1930), 19-27.

28. K. Prachar, Primzahlverteilung, Die Grundlehren der Math. Wiss., Vol. 91, SpringerVerlag, Berlin, 1957.

29. E. Schmidt, Math. Ann. 57 (1903), 195-204.

30. P. L. Tchebycheff, Bull. de la Classe Phys., Math. de l'Acad. Imperiale des Sciences, St. Petersbourg 11 (1853), 208.

31. E. C. Titchmarsh, The theory of the Riemann zeta function, The Clarendon Press, Oxford, 1951.

32. P. Turán, Eine neue Methode in der Analysis und deren Anwendungen, Akademiai Kiado, Budapest, 1953.

University of Pennsylvania,

Philadelphia, Pennsylvania 